\title{
EL USO DEL INTERNET COMO HERRAMIENTA PARA POTENCIAR LA MATRIZ PRODUCTIVA DE LA INDUSTRIA DEL CAMARÓN EN ECUADOR
}

\author{
THE USE OF THE INTERNET AS A TOOL TO STRENGTHEN THE PRODUCTIVE \\ MATRIX OF THE SHRIMP INDUSTRY IN ECUADOR
}

Edwin G. Cóndor G., ${ }^{1}$ Miguel A. Bustamante U., ${ }^{2 *}$

1 Universidad Católica de Santiago de Guayaquil, Ecuador. Av. Carlos Julio Arosemena Km. 1¹⁄2 vía Daule, Guayaquil, Ecuador. (e-mail: condor.edwin@ hotmail.com)

2 Facultad de Economía y Negocios, Universidad de Talca, 2 Norte 685, Talca, Chile.

RESUMEN

ABSTRACT

El presente trabajo analiza el uso de las herramientas de internet en la industria del camarón ecuatoriano, a fin de determinar cómo esta puede mejorar sus niveles de producción y eficiencia. Se aplicó un cuestionario estructurado en cuatro secciones que abordan geografía, demografía, aspectos sicográficos y conductuales a una muestra de directivos de 165 empresas del sector camaronero, para indagar en torno al uso de la tecnología en sus diversas dimensiones. Como resultado, se obtiene que únicamente el $4 \%$ de las empresas consideran internet como una herramienta clave en las actividades que desarrollan, la velocidad de conexión está por debajo de las recomendaciones internacionales y el $73 \%$ de las empresas no disponen de un portal web. Se concluye finalmente que la industria camaronera ecuatoriana está lejos de alcanzar las velocidades de acceso a Internet recomendadas, sin embargo, se observa que el objetivo es precisamente alcanzar la mayor automatización para alcanzar, efectivamente, ventajas competitivas en la industria.

PALABRAS CLAVE: Internet, productividad, eficiencia, competitividad, matriz productiva.
The present work analyzes the use of internet tools in the Ecuadorian shrimp industry, in order to determine how this can improve its production and efficiency levels. A structured questionnaire was applied in four sections dealing with geography, demography, psychographic and behavioral aspects to a sample of executives from 165 companies in the shrimp sector, to investigate the use of technology in its various dimensions. As a result, only $4 \%$ of companies consider the internet as a key tool in the activities they develop, the Internet access connection is below international recommendations and $73 \%$ of companies do not have a website. It is finally concluded that the Ecuadorian shrimp industry is far from reaching the recommended Internet access connection; however, it is observed that the objective is precisely to achieve the highest automation to achieve, effectively and competitive advantages in the industry.

KEY WORDS: Internet, productivity, efficiency, competitiveness, productive structure. 


\section{Introducción}

En enero del año 2017 el 50\% de la población mundial accedió a Internet y se espera que esta taza incremente a $51,5 \%$ en 2019 (Statista, 2017; Baller, et al., 2016) y se espera que para el año 2022, 29 billones de máquinas se encuentren interconectados a través de internet (Erickson, 2017).

Ecuador no es la excepción a esta tendencia, la densidad de usuarios con acceso fijo a Internet incrementó de $3,35 \%$ en 2010 a $9,76 \%$ en 2016, con acceso a Internet móvil incrementó de $2,35 \%$ en 2010 a $47,04 \%$ en 2016 (Agencia de Regulación y Control de las Telecomunicaciones, 2017). Por otra parte, de acuerdo con lo analizado por el Ministerio de Telecomunicaciones y Tecnologías de la Información (2016), se prevé que para el año 2021 la penetración de acceso a Internet mediante conexiones de banda ancha fija pase de $33 \%$ el año 2016 a 59\% el 2021 y las conexiones de acceso a Internet mediante conexiones de banda ancha móvil pasen del 31\% el año 2014 a 64\% el 2021.

La densidad de estas innovaciones macro sociales permiten prever incrementos en el potencial, también a nivel de la industria local, generando un potencial de optimización en los procesos de producción e incluso, el desarrollo de una industria sostenible en lo social y con el medio ambiente (Comisión Económica para América Latina y el Caribe, 2016aㅗ ttle, 2017; Organización de las Naciones Unidas para el desarrollo Industrial, 2017; PWC, 2017). En esta misma línea, la constitución del Ecuador (2008) y el Plan del Buen Vivir (20132017) (Secretaria Nacional de Planificación y Desarrollo, 2017), proponen un plan estructurado de cambio de su matriz productiva, sin embargo no existen parámetros claros o guías a seguir en cuanto a la utilización del Internet como medio para alcanzar este objetivo.

\section{Contextualización}

Para ilustrar, a nivel mundial, los alimentos acuáticos son de los productos alimenticios más comercializados alcanzando más allá del $50 \%$ de las exportaciones, que en general provienen de países en vías en desarrollo, tales como Ecuador (Food and Agriculture Organization, 2016). De hecho, la industria del camarón ecuatoriano tiene una tasa de crecimiento de $6 \%$ en promedio en los últimos cinco años (Cámara Nacional de Acuacultura, 2017).

El Ecuador es un país de América del Sur que se encuentra ubicado en la mitad del mundo. Limita al Norte con Colombia, al sur y al este con Perú y al oeste con el océano Pacífico. Ecuador es el octavo país más grande de América del Sur. Posee un área de $283.561 \mathrm{~km} 2$ y una población que supera los 16 millones de habitantes, lo que transforma en el octavo país más poblado de Latinoamérica (Mateo, 2015; Burque, 2015).

Dentro de las principales áreas de ingreso del Ecuador se pueden denotar la exportación de materias primas como petróleo, banano, camarón, cacao y rosas (Ekos, 2015; CNA, 2017). Según el Banco Mundial (2017) la economía del Ecuador ocupa el puesto 62, con un PBI-PPA (producto interno bruto y paridad adquisitiva) de 185 mil millones de dólares, adicionalmente tiene la séptima economía más grande de Sudamérica. Complementariamente y conforme los análisis del World Economic Forum (2017), se reporta que Ecuador ocupa el puesto 91 de competitividad de entre 138 países, en tanto que algunos países vecinos tales como Colombia y Perú, ocupan los puestos 61 y 67 respectivamente.

El Ecuador ha estado viviendo cambios políticos desde el año 2006. Durante este periodo han existido mejoras en la innovación vial (Ministerio de Transporte y Obras Publicas, 2017), pero no se han establecido políticas claras que fomenten la generación de riqueza sostenible, y permitan potenciar y expandir fuentes sostenibles de riqueza que permitan disponer al país de ingresos a corto y largo plazo, con el objetivo fundamental de comenzar a ser un país desarrollado (Friedrich-Ebert-Stiftung Ecuador, 2016; Burque, 2015).

En los últimos años, se ha comenzado a dar pequeños pasos en el cambio de la matriz productiva, mismo que permitiría al país, transformar su actual estructura industrial, desde la venta de materias primas hacia la generación de valor agregado (Ekos, 2015; FES-ILDIS, 2016; SENPLADES, 2017) acción que se ha potenciado en los últimos diez años, permitiendo al país niveles superiores de exportación (SENPLADES, 2017), considerando que el mundo está entrando en una cuarta revolución industrial (Baller, et al., 2016).

Finalmente, de acuerdo con lo analizado, el presente trabajo busca analizar el uso de las herramientas de internet en la industria del camarón ecuatoriano, a fin de determinar cómo esta puede mejorar sus niveles de producción y eficiencia.

\section{Innovación tecnológica}

CEPAL (2016a), indica que el uso del internet ha pasado a convertirse en una herramienta de producción, en tanto permite potenciar sectores estratégicos de generación de riqueza tales como: educación, investigación, comercio, salud y gobierno (CEPAL, 2016b), concordando con los lineamientos de organismos internacionales respecto de las Tecnologías de Información (MINTEL, 2016).

Por una parte, porque la productividad está influenciada por tecnología, la innovación y la elaboración de productos y procesos (ONUDI, 
2017; Boston Consulting Group, 2015) que contribuyen a la expansión y a la innovación para fortalecer las ventajas competitivas (Mateo, 2015; Little, 2017; BCG , 2015; Erickson, 2017), impactando los patrones de crecimiento económico y sostenibilidad de los países (CEPAL, 2016a, ONUDI, 2017, y Baller, Dutta y Lanvin, 2016).

\section{Modelos económicos de análisis}

El modelo de desarrollo a través de la matriz productiva (Burque, 2015) depende de la evolución técnica y humana de las fuerzas productivas, las que ha sido analizadas mediante los enfoques ortodoxos y heterodoxos complementarios (Siegle, 2017; Romo, 2017).

Por una parte, Siegle (2017) asociando la economía ortodoxa a la economía convencional de Ricardo, Mark y Keynes (Lima, Amado, \& Mollo, 2017) que sigue las reglas de la asignación de los recursos mediante mercados eficientes (Siegle. 2017; Romo, 2017; Lima, Amado, \& Mollo, 2017).

Sin embargo, los sectores económicos son diversos en sus procesos (Burque, 2015; BCG, 2015) ajustándose a sus respectivos contextos y regulaciones del Estado (ONUDI, 2017; Burque, 2015) para fortalecer la matriz productiva (Burque, 2015; Ekos, 2015; FES-ILDIS, 2016). Sin embargo el modelo heterodoxo (Siegle, 2017; Lima, et al., 2017) se contrapone con la economía ortodoxa (Siegle, 2017; Romo, 2017) que más bien busca sustituir el modelo extractivo por la producción y consumo de bienes con mayor valor agregado mediante la industrialización (ONUDI, 2017; FES-ILDIS, 2016; GTAI, 2017, BCG, 2015).

\section{La cuarta revolución industrial}

CEPAL (2016a), CEPAL (2016b) y ONUDI (2017) sostienen que en los años 80 el desarrollo de la electrónica, las computadoras y el Internet abrieron el portal a un cambio tecnológico nunca antes visto. BCG (2015), PWC (2017) y The Little (2017) añaden que el desarrollo tecnológico que estamos viviendo, nos abre la puerta a una nueva revolución, la misma que sobrepasara las vivencias de las revoluciones anteriores. Por su parte $\mathrm{Ba}$ - ller, Dutta, y Lanvin (2016) sostiene que cada vez el número de usuarios que navegan en Internet crece de una manera exponencial.

Se prevé que para el 2022 se conectaran al Internet más de 29 billones de dispositivos y se tendrá más de 4 mil millones de usuarios de Internet a nivel Global (Baller, Dutta y Lanvin, 2016; BCG, 2015; Ericson, 2017; Little, 2017) concuerdan en que esta demanda exponencial del uso de Internet ha llevado al mundo a entrar en su cuarta revolución industrial, llamada también transformación digital.

Todas estas ventajas que ofrece el uso del Internet en todos las actividades que interactúan en la industria, nos permitirá optimizar los procesos de producción y reducir los tiempos de producción, con lo cual se generaran nuevos modelos de negocio y brindar a la industria ventajas competitivas (GTAI, 2017; Little, 2017).

Sobre la base de lo enunciado, el objetivo de este estudio es evaluar cómo el uso de la herramienta internet ayuda a potenciar la matriz productiva de la industria del camarón.

\section{Metodología}

Para el desarrollo del presente trabajo se aplicó un diseño no experimental (Bruhn, 2015; Lopez \& Sandoval, 2016), transversal, descriptivo y cuantitativo (Lopez \& Sandoval, 2016), con el apoyo del método cualitativo (Herrera, 2016) en algunas de las fases de recolección de datos. Para la recogida de datos, se implementó el trabajo de campo para la aplicación de un cuestionario estructurado en secciones e ítems (Bruhn, 2015) a una muestra de 165 empresas del sector camaronero, para indagar en torno al uso de la tecnología digital, el uso del Internet y el eventual impacto en los negocios (Arellano y Peralta, 2015; OECD, 2015; Statistics Canada, 2016).

Se tuvo en consideración la segmentación del mercado (Echeverría, 2017), tomando en consideraciónlas variablesqueseindican enlaTabla1.

Tabla 1 Variables de Segmentación

\begin{tabular}{ll}
\hline \multicolumn{1}{c}{ Variable } & \multicolumn{1}{c}{ Segmento } \\
\hline Geográfica & $\begin{array}{l}\text { El proyecto se enfocará en la región costa ecuatoriana. } \\
\text { Provincias: El Oro, Guayas, Manabí, Santa Elena. }\end{array}$ \\
\hline Demográfica: & $\begin{array}{l}\text { Empresas Camaroneras ubicadas en las provincias de El Oro, Guayas, Manabí, Santa Elena, } \\
\text { Pichincha, Esmeraldas, Bahía de Caraquez. }\end{array}$ \\
\hline Sico gráfica: & Identificar el uso actual del Internet en las actividades involucradas en la industria del camarón. \\
\hline Conductual: & $\begin{array}{l}\text { Motivos y decisiones de utilización del Internet en todas las actividades que intervienen en la } \\
\text { cadena de valor en la industria del camarón }\end{array}$ \\
\hline
\end{tabular}




\section{Procedimiento de muestreo}

Para la aplicación de los procedimientos más adecuados a la investigación se elaboró un muestreo a partir del registro oficial del listado de las empresas camaroneras que son miembros del CNA (2017), que en total son 219 empresas. Para calcular la muestra se aplicó la "fórmula para poblaciones finitas" (Herrera, 2016), por lo tanto, el cálculo quedó definido por formula (1).

$$
\mathbf{n}=\frac{\mathbf{Z}^{2 *} \mathbf{N}^{*} \mathbf{p}^{*} \mathbf{q}}{\mathbf{e}^{2 *}(\mathbf{N}-\mathbf{1})+\mathbf{Z}^{2 *} \mathbf{p}^{*} \mathbf{q}}
$$

Dónde:

$\mathrm{n}=$ Tamaño de la muestra

$\mathrm{Z} 2$ = Nivel de confianza $96 \%=2.05$

$\mathrm{N}$ = Tamaño de la población $=219$ empresas

$\mathrm{p}=$ Probabilidad de aceptación $50 \%=0.50$

$\mathrm{q}=$ Probabilidad de no aceptación $50 \%=0.50$

e2 $=$ Margen de error permitido $=0.04$

Aplicada la formula se obtuvo el siguiente resultado en (2):

$\mathrm{n}=\frac{2,052^{*} 219^{*} 0,5^{*} 0,5}{0,0042^{*}(219-1)+2,052^{*} 0,5^{*} 0,5}=164,41$
Por lo tanto se aplicaron 165 cuestionarios, en la ciudad de Quito y Guayaquil, mediante un trabajo de campo que se concretó a finales del año 2017. Para ello se contactó a Gerentes y/o encargados de las áreas de desarrollo de proyectos, ventas y marketing de las empresas seleccionadas.

\section{Resultados}

A continuación se presenta una síntesis de la disponibilidad de la tecnología a nivel global y la que se logró determinar para la realidad de la industria camaronera ecuatoriana.

El uso de internet a nivel global

Baller, Dutta, \& Lanvin, 2016; CEPAL, $2016^{\mathfrak{a}}$; y ONUDI, 2017, argumentan que la utilización masiva de Internet mediante conexiones de banda ancha en todos los elementos que conforman la sociedad, impactan en los patrones de crecimiento económico, la sostenibilidad y la inclusión social de los países (Tabla 2, Adaptada de Zhuang, et al., 2013).

Tabla 2 Ancho de banda requerido según el tipo de aplicaciones en Mbps

\begin{tabular}{lc}
\hline Proyectos Mozilla Ignite y US Ignite Ancho de banda requerido & Rango \\
\hline Manufactura avanzada & 38 a 74 Mbps \\
Preparación para emergencias y seguridad & 6 a 18 \\
Educación y capacitación & 38 a 74 Mbps \\
Tecnologías de la salud & 38 a 74 Mbps \\
Redes limpias de energía y transporte & 2 a 3 Mbps \\
Monitoreo de clima y aviones & 38 a 74 Mbps \\
Uso de video interactivo en 3D & 77 a 148 Mbps \\
\hline
\end{tabular}

\section{Penetración del Internet a Nivel Mun-} dial

ITU (2016) reporta que el $53 \%$ de la población a nivel mundial aún no utiliza Internet y de la parte que lo utiliza, el $86 \%$ de las casas en países desarrollados dispone de una conexión a Internet en comparación al 41,1\% en países en vías de desarrollo y al $11.1 \%$ en países menos desarrollados. En relación a la velocidad de acceso, Akamai (2017) e ITU (2016) concuerdan en que existe una gran diferencia en cuanto a las velocidades de acceso a Internet entre países desarrollados y países en vías de desarrollo como se muestra en la Tabla 3 (Adaptado de Akamai, 2017, p.14).

Tabla 3 Velocidad en promedio de acceso a Internet mediante conexiones de banda ancha (IPV4) por país.

\begin{tabular}{ccc}
\hline Clobal Rank & Country/Región & Q1/2017 Avg. Mbps \\
\hline- & Clobal & 7.2 \\
1 & Corea del Sur & 28.6 \\
2 & Noruega & 23.5 \\
3 & Suecia & 22.5 \\
4 & Hong Kong & 21.9 \\
5 & Suiza & 21.7 \\
6 & Finlandia & 20.5 \\
7 & Singapur & 20.3 \\
8 & Japón & 20.2 \\
9 & Dinamarca & 20.1 \\
10 & Estados Unidos & 18.7 \\
\hline
\end{tabular}


En síntesis, más del 50\% de la población a nivel mundial aún no tiene la oportunidad de acceder a servicios de Internet. Adicional, existe una gran brecha en el uso de Internet entre países desarrollados y vías de desarrollo parte del problema es la diferencia de costo de los servicios de tecnologías digitales entre países desarrollados y países en vías de desarrollo como el Ecuador.

\section{Análisis descriptivo del uso de Inter- net a nivel ecuatoriano en la industria camaronera}

Se observa que las empresas Camaroneras Ecuatorianas en su mayoría son pequeñas con un $44 \%$. Le siguen las empresas medianas y grandes con un porcentaje de $28 \%$. Se observa además, que las empresas Camaroneras que tienen una permanencia en el mercado mayor a diez años ocupan el $64 \%$ de la muestra tomada. Lo siguen las empresas con un periodo de permanencia de cinco a diez años con un $24 \%$ y finalmente se tienen las empresas más jóvenes con un $12 \%$, observándose madurez en el sector Camaronero. Sin embargo, es de suma importancia tener en mente que únicamente el $5 \%$ del total de empresas encuestadas no dispone de una conexión a Internet.

Tabla 4 Tabulación de datos tiempo constitución empresa

\begin{tabular}{lclc}
\hline Tiempo de constitución de la empresa & Total & Tamaño de la empresa & Total \\
\hline Menor a 5 años & 19 & Pequeña (de 1 a 49 colaboradores) & 72 \\
De 5 a 10 años & 40 & Mediana (de 50 a 99 colaboradores) & 46 \\
mayor a 10 años & 106 & Crande (mayor a 100 colaboradores) & 47 \\
Total & 165 & Total & 165 \\
\hline \multicolumn{5}{c}{ Dispone de conexión a Internet } \\
\hline \\
$\mathrm{Si}$ & & 157 & \\
& & 8 & 165 \\
\hline
\end{tabular}

Se observa por otra parte, que la conexión a Internet mediante Cable modem es el preferido de las empresas Camaroneras con un 56\% de preferencia. Lo siguen las conexiones mediante acceso Inalámbrico Celular con un $36 \%$. A continuación se tienen las conexiones a Internet mediante conexión de Fibra Óptica con un $12 \%$, seguido de conexiones a Internet mediante Radio enlace con $7 \%$, seguido de conexiones satelital para salir a Internet con un $6 \%$

Finalmente, se observa que existe un bajo porcentaje de empresas que desconoce el tipo de conexión dispone para salir a Internet. Estos resultados concuerdan con las tendencias de la industria (Erickson, 2017) y con las estadísticas mostradas por Arcotel (2017), los cuales muestran que la conexión a Internet mediante medios celulares como $3 \mathrm{G}$ y $4 \mathrm{C}$ están incrementándose en los últimos años.

De las 157 empresas que reportaron que sí disponen de una conexión a Internet (Tabla 4), se observa que el $73 \%$ del total de empresas no dispone de una página web, de los cuales el $36 \%$ corresponde a empresas pequeñas, el $23 \%$ a empresas medianas y el $14 \%$ a empresas grandes. Por otra parte, el $27 \%$ del total de empresas dispone de una página web, de las cuales el 5\% corresponde a empresas pequeñas, el $6 \%$ a empresas medianas y el $15 \%$ a empresas grandes.

De las 42 empresas que reportaron disponer de una página web (Tabla 5), las mismas que disponen en un $17 \%$ de la característica de permitir ventas a través de Internet, el $48 \%$ es un portal seguro, el $10 \%$ es un portal que permite recolectar y analizar en tiempo real la información de los clientes, el $26 \%$ tiene una integración total con medios digitales, el $55 \%$ permite rastrear los activos fijos de la empresa y el $14 \%$ es un portal estático.

Respecto de la velocidad de acceso a Internet (Tabla 5), el 24\% del total de la muestra corresponde a pequeñas empresas, disponen de una conexión a Internet menor a cinco Mbps, lo siguen las empresas medianas con un $17 \%$ y finalmente las empresas grandes con el $4 \%$.

En relación a las empresas que disponen de una conexión a Internet con una velocidad de acceso a Internet de entre cinco a diez Mbps, el $11 \%$ corresponde a pequeñas empresas, lo siguen las empresas medianas con un $8 \%$ y finalmente las empresas grandes con el $12 \%$. Por otro lado, las empresas que reportaron disponer de una conexión a Internet con una velocidad de acceso mayor a diez Mbps, el $1 \%$ corresponde a pequeñas empresas, lo siguen las empresas medianas con un $1 \%$ y finalmente las empresas grandes con el $4 \%$.

Es importante tomar en cuenta que, con la aparición de nuevas tecnologías como es el Internet de las cosas, las tecnologías celulares deberían ser de las más recomendadas para potenciar la cadena de valor de la industria.

Finalmente, las empresas que reportaron no conocer el tipo de conexión a Internet que disponen (Tabla 5), el 5\% corresponde a pequeñas empresas, lo siguen las empresas medianas con un $4 \%$ y al final las empresas grandes con el $9 \%$. 
Tabla 5 Tecnología de internet y efectividad de negocio

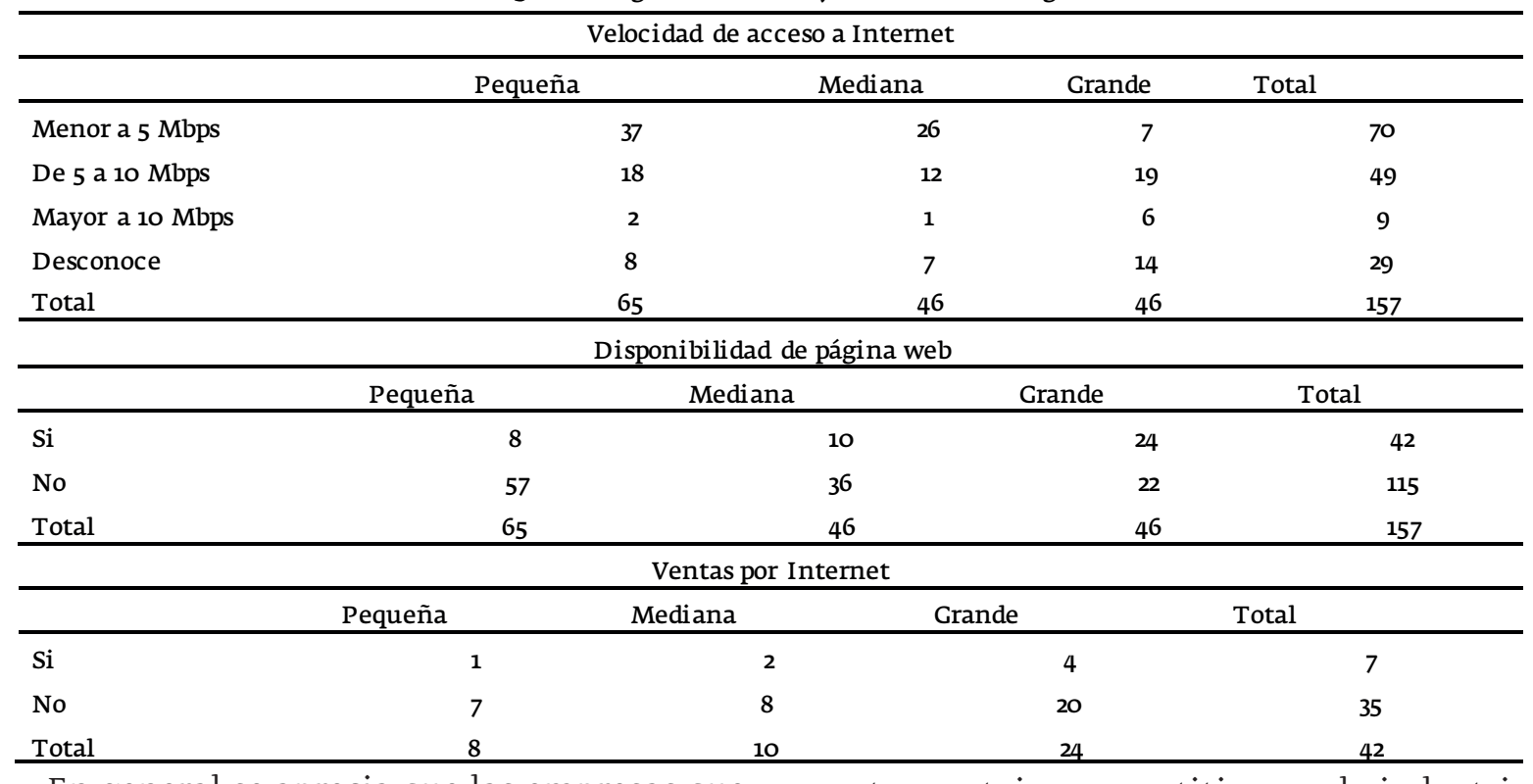

En general se aprecia que las empresas que disponen de una conexión a Internet con una velocidad de acceso menor a cinco Mbps corresponde al $45 \%$, seguido de las empresas con una velocidad de acceso de entre cinco y diez Mbps que son el $31 \%$, seguido de las empresas que disponen de una conexión de acceso a Internet mayor a diez Mbps que corresponden al $6 \%$. Dichos valores se encuentran muy por debajo de la velocidad sugerida en la industria manufacturera que se encuentra en un rango de 38 a 74 Mbps como se muestra en la Tabla 2. Finalmente, el 18\% de las empresas desconoce la velocidad de acceso a Internet que disponen, lo cual tiene relación con la poca importancia que las empresas le brindan al Internet, ya que únicamente el $4 \%$ de las empresas consideran al Internet como una herramienta clave para potenciar las actividades que realizan.

\section{A modo de síntesis}

En general, las empresas Camaroneras en Ecuador no están haciendo uso de las ventajas que brindan las tecnologías digitales. Es imperativo, en consecuencia, atacar esta falla en infraestructura tecnológica que actualmente se tiene para que la industria del Camarón tenga ventajas competitivas frente al resto de países, mejore su visibilidad de negocios internacionales y se proyecte al mundo como una industria sostenible que ha encontrado en las tecnologías digitales como el acceso a Internet su punta de lanza para convertirse en una industria sostenible y generadora de riqueza.

Es claro, en consecuencia, que la industria Ecuatoriana se encuentra lejana de alcanzar las velocidades de acceso a Internet recomendadas por la industria, sin embargo, se observa que el objetivo es precisamente alcanzar la mayor automatización para alcanzar, efectiva- mente, ventajas competitivas en la industria.

Por lo tanto, es claro que aun cuando existan empresas que disponen de una página web, los sitios no disponen de todas las ventajas que brindan las tecnologías digitales, los mismos que brindan una ventaja competitiva a las empresas con el objetivo de brindar un mayor valor agregado a los clientes.

\section{Conclusiones}

La utilización de la herramienta Internet mediante el uso de tecnologías de banda ancha, permite a la industria profundizar en las actividades primarias y en las actividades de apoyo dentro de la cadena de valor con el objetivo de mejorar su productividad e incrementar sus resultados.

En general, los países de Latino América $y$ en particular Ecuador, no han tomado de una manera adecuada el uso de Internet como una herramienta clave para su crecimiento y desarrollo.

Solo un bajo porcentaje de las empresas camaroneras Ecuatorianas dispone de una velocidad de conexión a Internet superior y, a nivel similar, algunas pocas empresas camaroneras ha considerado Internet como herramienta clave dentro de las actividades que realiza.

La mayoría de las empresas camaroneras no dispone de un portal web, ubicándose de espalda a las ventajas que brinda el uso del Internet, sin incorporar las ventajas de la velocidad de acceso ni las opciones que les reportaría el disponer de un portal web interactivo.

\section{Referencias}

Akamai. (2017). akamai's [state of the internet] Q1 2017 report. Disponible en: https://www.akamai.com/us/ en/about/our-thinking/state-of-the-internet-report/ 
global-state-of-the-internet-connectivity-reports.jsp ARCOTEL (Agencia Nacional de Regulación y Control de las Telecomunicaciones). (2017). Boletín Estadístico Agencia de Regulación y Control de las Telecomunicaciones - IV Trimestre de 2016.

Arellano, P. Peralta, S. (2015). Informe de resultados: Tecnologias de la Información y comunicación en las empresas.

Baller, S., Dutta, S. , \&Lanvin, B. (2016). The globalinformation technology report 2016 World Economic Forum, Geneva.

BCG (The Boston Consulting Group). (2015). Industry 4.0 the future of productivity and growth in manufacturing in dustries. Disponibleen:https://www.zvw.de/media.media.72e472fb-1698-4a15-8858-344351c8902f. original.pdf

BM (Banco Mundial). (2017). PIB, PPA ( $\$$ a precios internacionales actuales). Disponible en: http://datos. bancomundial.org/indicador/NY.GDP.MKTP.PP.CD

Burque, S. (2015). ¿ Cómo afecta la matriz productiva 2014 a los productos primarios en el Ecuador (petróleo, banano, camarón, cacao)? (Bachelor's thesis, Quito: USFQ, 2015).

Bruhn, K. (2015). La Comunicación y los medios. Metodologias de investigacion cualitativa y cuantitativa.

CEPAL (Comisión Económica para América Latina y el Caribe) (2016a). La nueva revolución digital de la Internet de consumo a la Internet de producción.

CEPAL (Comisión Económica para América Latina y el Caribe) (2016b). El cambio tecnológico y el nuevo contexto del empleo Tendencias generales y en América Latina.

CNA (Cámara Nacionalde Acuacultura).(2017). Exportaciones de camarones de 2000 a 2016 (ENE-DIC) Libras vs. Dolares .

Echeverría, C. (2017). Metodología para determinar la factibilidad de un proyecto.

Ekos (2015). Producto ecuatoriano y cambio de Matriz Productiva. En Ekos Negocios.

Erickson (2017). Ericsson mobility report.

FAO (Food and Agricultur Organization). (2016). EL ESTADO MUNDIAL DE LA PESCA Y LA ACUICULTURA. Contribución a la seguridad Alimentaria y la nutrición para todos.

FES-ILDIS (Friedrich-Ebert-Stiftung Ecuador). (2016). BUEN VIVIR Y CAMBIO DE LA MATRIZ PRO DUCTIVA Reflexiones desde el Ecuador. Quito: Editorial de la Universidad de Cantabria.

GTAI (Germany Trade \& Investment), (2017). Industry 4.0 Smart Manufacturing for the future. Disponible en:

Herrera, J. (2016). La investigación cualitativa ITU (International Telecommunication Union). (2016). ICT 2016 Facts and Figures 2016.
Lima, P., Amado, A., \& Mollo, M. (2017). Fundamentos macroeconômicos nas perspectivas de Marx e Keynes : contribuições para a heterodoxia. Little, A. (2017). The German Internet Industry. Lopez, N., Sandoval, I (2016). Fundamentos macroeconômicos nas perspectivas de Marx e Keynes : contribuiçôes para a heterodoxia. Mateo, B. (2015). Estudio del comercio electrónico como estrategia de negocios dentro de las empresas que brindan servicios de hospedaje en el Cantón Salinas, Provincia de Santa Elena. MINTEL(MinisteriodeTelecomunicaciones yde Sociedad dela Información) (2016). Plan Nacional de Telecomunicaciones yTecnologías dela Información del Ecuador 2016-2021.

MTOP (Ministerio de Transporte y Obras Públicas). (2017). PLAN ESTRATEGICO DE MOVILIDAD 2013 - 2037.

OECD (Organización para la cooperación y el desarrollo económico) (2015). The OECD Model Survey on ICT Access and Usage by Households and Individuals.

ONUDI (Organización de las Naciones Unidas para el desarrollo Industrial). (2017). Informe sobre el desarrollo Industrial 2016. El rol de la tecnología y la innovación en el desarrollo industrial inclusivo y sostenible.

PWC. (2017). The Digital Future of creative Europe. The impact of digitization and the Internet on the creative industries in Europe.

Romo, C. (2017). El género dentro de la cinta de Moebius de los modelos de desarrollo.

SENPLADES (Secretaria Nacional de Planificación y Desarrollo). (2017). buen vivir plan nacional 2013-2017.

Siegle, D. (2017). Ortodoxos e heterodoxos: uma análise do debate através de diferentes metodologias de pesquisa.

Statista (2017). Global internet penetration rate as of January 2017, by region. Disponible en: https://www.statista.com/statistics/269329/penetration-rate-of-the-internet-by-region/

Statistics Canada (2016). Survey of Digital Technology and Internet Use (SDTIU). Disponible en: http://www23.statcan.gc.ca/imdb-bmdi/instrument/4225_Q1 V1o-eng.pdf

World Economic Forum (2017). The Global Competitiveness Report.

Zhuang, Y., Cappos, J., Rappaport, T., \& McGeer, R. (2013). Future Internet Bandwidth Trends: An Investigation on Current and Future Disruptive Technologies. Tech. rep. tr-cse-2013-04, Polytechnic Institute of NYU. Disponible en: https://ssl. engineering.nyu.edu/papers/tr-cse-2013-04.pdf 